NASA Technical Memorandum 107573

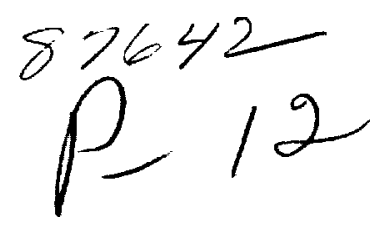

\title{
UNDERLYING MODAL DATA ISSUES FOR DETECTING DAMAGE IN TRUSS STRUCTURES
}

Thomas A-L. Kashangaki, Suzanne Weaver Smith, and Tae W. Lim

February 1992

\section{N/Sก \\ National Aeronautics and \\ Space Administration \\ Langley Research Center \\ Hampton, Virginia 23665}

(NASA-TM-107573) UNDERLYING MOOAL DATA

N92-23990

ISSUES FOR DETECTING DAMAGE IN TRUSS

STRUCTURES (NASA) $12 \mathrm{p}$ CSCL 2OK 
$=\cdots$ 


\author{
Thomas A-L Kashangaki * \\ NASA Langley Research Center \\ Hampton, Virginia \\ Suzanne Weaver Smith ${ }^{+}$ \\ University of Kentucky \\ Lexington, Kentucky \\ Tac W. Lim \# \\ Lockheed Engineering and Sciences Company \\ Hampton, Virginia
}

\begin{abstract}
Independent of the modal identification techniques employed for damage detection, use of measured modal data limits the abilit) to detect damage. These limitations are examined using the distribution of modal strain energy and the sensitivity of the frequency and mode shapes to structural stiffness changes. For given measured modal information of specified accuracy, this examination reveals the following: (1) damage detection is feasible for members that contribute significantly to the strain energy of the measured modes, (2) the modes which are most effective in detecting damage to certain critical members can be identified and (3) a relationship can be drawn between the accuracy of the measured modes and frequencies and damage detection feasibility.
\end{abstract}

\section{Introduction}

The complexity and size of proposed space structures, along with the investment they represent,' require that the integrity of the structures be monitored periodically. Researchers have suggested that system identification techniques which use modal properties measured from the structure might be useful for structural integrity verification and damage location 1,3,4,7. To understand the extent to which this approach could be useful, underlying issues which involve the measured modal properties are examined in this paper.

Independent of the system identification technique used, if damage location is to be accomplished through the use of modal properties measured from the structure, the damage must affect the response of the structure so that the effect is observable and measurable in the modal properties.

\footnotetext{
* Acrospace Engineer, Member AIAA

+ Assistant Professor, Dept. of Engineering Mechanics, Member AIAA

\# Senior Engineer, Member AIAA
}

Even if a damaged element impacts the modal properties of the lower frequency modes, this impact must exceed the levels of uncertainty in modelling or testing which are present, so that the damaged element of the truss can be located and then replaced or repaired.

In this paper, to explore the limitations that are present with the use of modal data, strain energy distributions, [rcquency sensitivities and mode shape variations are studied for an eight bay laboratory truss structure and expectations for the ability to locate damage are discussed.

\section{Backeround}

This research is part of an ongoing effort to examine damage detection for large, flexible space truss structures. Figure 1 shows the eight bay hybrid-scaled truss which was introduced in a previous work ${ }^{1}$ as a focus structure for this damage detection research. This truss is one component of a scale model of an early Space Station Freedom configuration. The eight bay truss was cantilevered from a 
wall and instrumented with 96 accelerometers to measure the three translational degrees of freedom at each node.

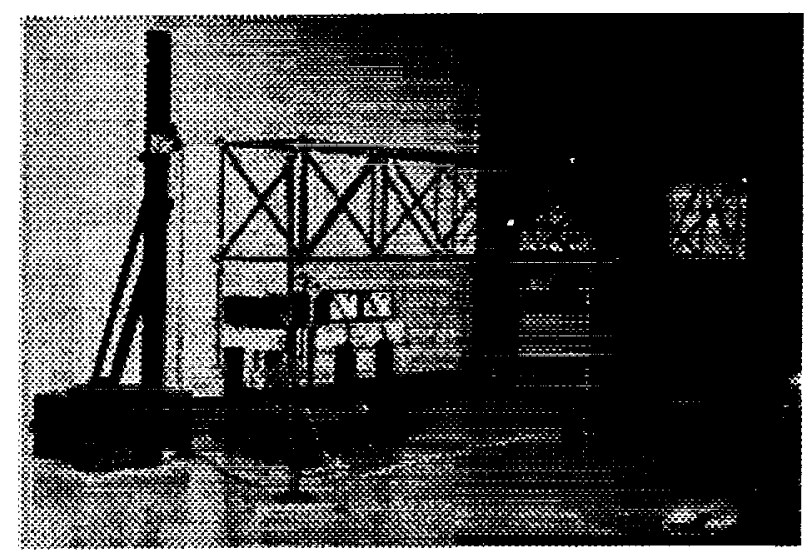

Figure 1. Eight bay test structure

Three excitation sources were used and mode shapes and frequencies extracted using the Polyreference complex exponential technique developed by Vold et. al ${ }^{2}$. The first six modes of the structure were targeted for identification through modal testing. Local modes of the truss members occur in a frequency band above the sixth mode and complicate testing and identification for higher-frequency global modes. The six modes include two bending mode pairs, a torsional mode and an axial mode.

Figure 2 shows a finite element model of the truss with the damage cases that have been examined. Table 1 shows the type of element and the bay location of the damaged member for each damage case. Damage cases $\mathrm{H}, \mathrm{I}$ and $\mathrm{F}$ will be used as examples throughout the following.

Table 1. Ejght bay truss damage cases

\begin{tabular}{|c|c|c|c|}
\hline CASE & $\begin{array}{c}\text { ELEMENT } \\
\text { LABEL }\end{array}$ & $\begin{array}{c}\text { ELEMENT } \\
\text { TYPE }\end{array}$ & BAY \\
\hline A & 46 & Longeron & 1 \\
\hline B & 107 & Diagonal & 1 \\
\hline C & 39 & Longeron & 2 \\
\hline D & 102 & Diagonal & 2 \\
\hline E & 36 & X-Batten & 3 \\
\hline F & 75 & Diagonal & 3 \\
\hline G & 63 & Z-Batten & 3 \\
\hline H & 35 & Longeron & 3 \\
\hline I & 99 & Diagonal & 3 \\
\hline J & 28 & Longeron & 4 \\
\hline K & 92 & Diagonal & 5 \\
\hline L & 22 & Longeron & 5 \\
\hline M & 17 & Longeron & 6 \\
\hline N & 3 & Longeron & 8 \\
\hline O & 35 AND 99 & Longeron \& & 3 \\
& & Diagonal & \\
\hline
\end{tabular}

In the laboratory tests damage refers to removal of the strut, i.e., $100 \%$ loss of stiffness. In simulation studies, situations of less than $100 \%$ stiffness loss are also explored.

With the goveming equations for a finite-element model of an $n$ degree of freedom structural dynamic system written as

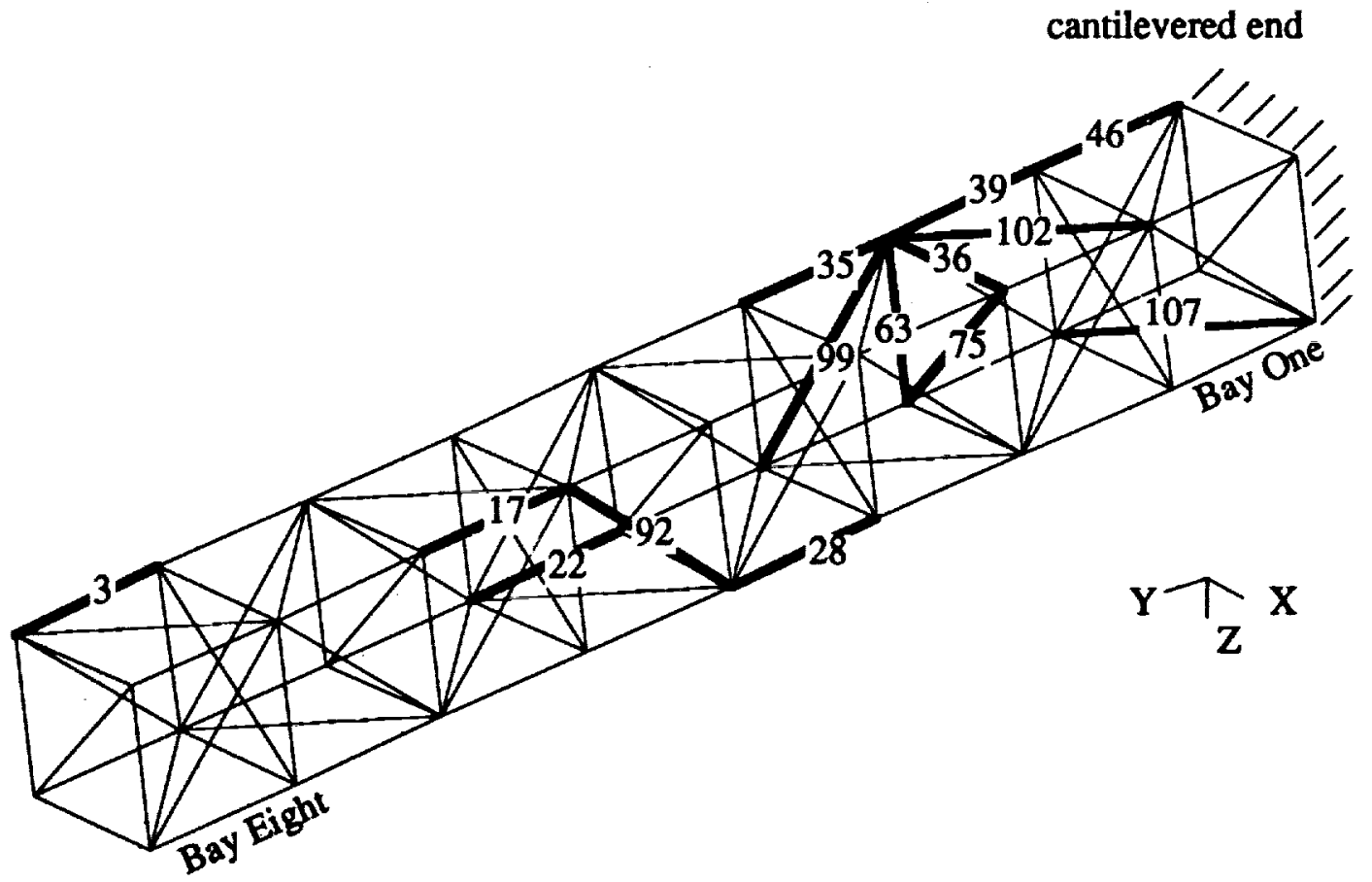

Figure 2. Eight bay truss damage cases

Page 2 


$$
[M]\{\ddot{x}\}+[D]\{\dot{x}\}+[K]\{x]=\{f(t)\}
$$

where

$[M]=n \times n$ mass matrix

$[D]=n \times n$ damping matrix

$[K]=n \times n$ stiffness matrix

$[x]^{"}=n x I$ vector of physical displacements and rotations at the nodes

$f(t)\}=n \times I$ vector containing the applied loads,

the undamped eigenvalue equation is

$$
\left([K]-\lambda_{i}[M]\right)\{\phi\}_{i}=0
$$

Solution of this equation yields a set of $n$ eigenvalue and eigenvector pairs, $\left[\lambda_{i}, \phi_{i}\right]$. Of these, only a few may be determined through tests of the structure. In the subsequent discussions, the mode shapes are scaled to be orthonormal with respect to the mass matrix. Therefore,

$$
\begin{gathered}
{[\Phi]^{T}[\boldsymbol{M}][\Phi]=[I]} \\
{[\Phi]^{T}[\boldsymbol{K}][\Phi]=[\lambda]}
\end{gathered}
$$

\section{Strain Energy as an Indicatoc of Ability le Detect Damage.}

Strain energy distribution has been used by previous researchers (see, for example, Refs. 1, 3 and 4) as an important measure in work related to structural identification. Hajela and Soiero ${ }^{3}$ used strain energy distributions and mode weighting factors to enhance the ability 10 detect damage. Here we presume that damage to a member will only be detected when the damage affects the modes measured in a test. Therefore a detailed study of the strain energy distribution was undertaken for the eight bay truss structure as an indicator of the relative participation of member $j$ in mode $i$. The strain energy distribution for the ith mode can be calculated as

$$
S E_{j i}=\frac{\{\phi\}_{i}^{T}\left[K_{j}\right]\{\phi\}_{i}}{\sum_{j=1}^{\dot{S}}\{\phi\}_{i}^{T}\left[K_{j}\right]\{\phi\}_{i}} \times 100 \%
$$

where $\left[K_{j}\right]$ is the stiffness submatrix of the jth element, and $e$ is the total number of stiffness elements in the model. This calculation is performed for each mode of interest $(i=1: m$ ) and each member $(j=1: e)$, resulting in $m$ columns of e percentage values. These columns are sorted in descending order. For each mode, the number of members that contribute significantly to the total strain energy is different. Higher modes include participation of a larger number of elements, but may be more difficult to identify from test, as in the case of the eight bay truss, in which local modes of the members interfere with the ability to identify higher frequency global modes of the structure.

Table 2 ranks the elements with the largest contributions to the total modal strain energy for each of the six lower modes. Numbers in parentheses are the percent strain energy for that element. Only members that contribute above $1 \%$ are listed. In each of these modes about $90 \%$ of the strain energy is represented by these members. Figure 3 presents the same information emphasizing that highly strained elements are near the root of the cantilevered truss.

Table 2. Highly strained members for modes 1 through 6

\begin{tabular}{lllllll} 
MODE 1 & MODE 2 & MODE 3 & MODE 4 & MODE 5 & MODE 6 \\
\hline $47(21)$ & $45(15.7)$ & $106(6.0)$ & $29(8.3)$ & $23(7.7)$ & $46(5.3)$ \\
$49(21)$ & $46(15.7)$ & $107(6.0)$ & $31(8.3)$ & $25(7.7)$ & $45(5.3)$ \\
$41(9.4)$ & $39(15.7)$ & $108(6.0)$ & $47(8.2)$ & $15(7.4)$ & $43(5.3)$ \\
$43(9.4)$ & $40(15.7)$ & $19(6.0)$ & $49(8.2)$ & $16(7.4)$ & $41(5.3)$ \\
$33(9.4)$ & $35(5.9)$ & $102(5.5)$ & $21(8.2)$ & $27(4.8)$ & $39(5.0)$ \\
$34(9.4)$ & $37(5.9)$ & $103(5.5)$ & $22(8.2)$ & $28(4.8)$ & $40(5.0)$ \\
$29(2.7)$ & $27(5.9)$ & $104(5.5)$ & $17(3.3)$ & $35(4.8)$ & $34(4.6)$ \\
$31(2.7)$ & $28(5.9)$ & $105(5.5)$ & $19(3.3)$ & $37(4.8)$ & $33(4.6)$ \\
$21(2.7)$ & $23(1.2)$ & $98(4.8)$ & $106(3.2)$ & $106(3.5)$ & $47(4.4)$ \\
$22(2.7)$ & $25(1.2)$ & $99(4.8)$ & $107(3.2)$ & $107(3.5)$ & $49(4.4)$ \\
& $15(1.2)$ & $100(4.8)$ & $108(3.2)$ & $108(3.5)$ & $37(4.2)$ \\
& $16(1.2)$ & $101(4.8)$ & $109(3.2)$ & $109(3.5)$ & $35(4.2)$ \\
& & $94(3.7)$ & $9(3.0)$ & $102(3.0)$ & $29(3.4)$ \\
& & $95(3.7)$ & $10(3.0)$ & $104(3.0)$ & $31(3.4)$ \\
& & $96(3.7)$ & $102(2.4)$ & $103(3.0)$ & $28(3.4)$ \\
& & $97(3.7)$ & $103(2.4)$ & $105(3.0)$ & $27(3.4)$ \\
& & $90(2.6)$ & $104(2.4)$ & $45(2.0)$ & $21(2.5)$ \\
& & $91(2.6)$ & $105(2.4)$ & $46(2.0)$ & $22(2.5)$ \\
& & $92(2.6)$ & $33(1.2)$ & $40(1.9)$ & $25(2.3)$ \\
& & $93(2.6)$ & $34(1.2)$ & $39(1.9)$ & $23(2.3)$ \\
& & $86(1.5)$ & $41(1.2)$ & $100(1.6)$ & $16(1.5)$ \\
& & $87(1.5)$ & $43(1.2)$ & $101(1.6)$ & $15(1.53$ \\
& & $88(1.5)$ & $98(1.1)$ & $98(1.6)$ & $17(1.4)$ \\
& & $89(1.5)$ & $99(1.1)$ & $99(1.6)$ & $19(1.4)$ \\
& & & $100(1.1)$ & & $76(1.1)$ \\
& & & & & \\
\hline
\end{tabular}

A pre-test strain energy analysis of a structure can be used to determine which modes must be identified in order to detect damage in critical members. For example, in order to detect damage in member 35 (damage case $\mathrm{H}$ ), Table 2 suggests that at least modes 2, 5 and 6 should be measured in the test. Modes 1,3 and 4 do not provide useful informaticn. Conversely, damage detection for a member that is not listed in the table would be difficult and additional instrumentation, for example strain gauges, would be required. 
Figure 4 indicates those elements which contribute to $50 \%$. $75 \%$, and $95 \%$ of the strain energy in all of the first six modes.
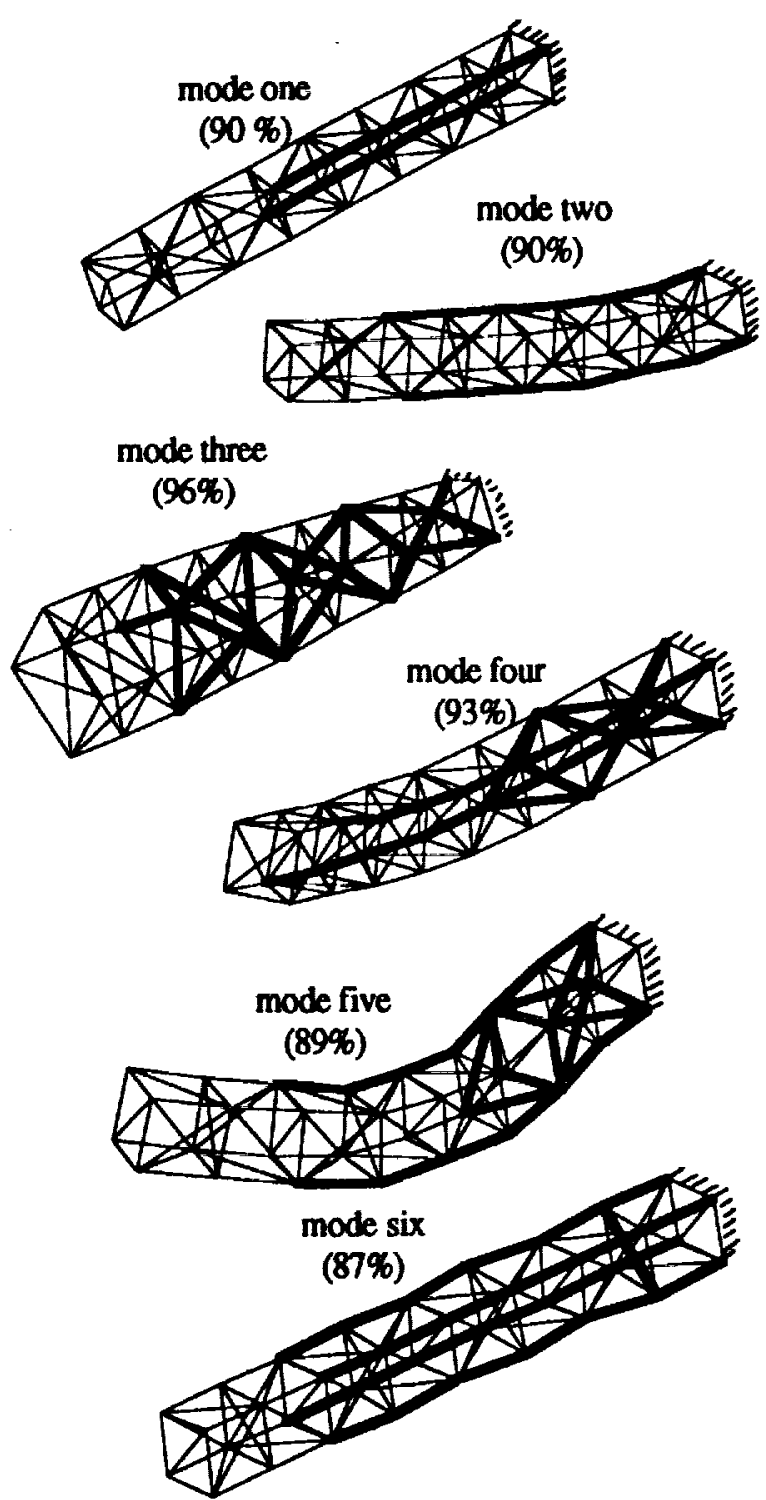

Figure 3. Highly strained members for modes 1 through 6

If strain energy distribution is indeed a good measure of the participation of a member in a mode, then from this figure it is apparent that it may be reasonable to expect to detect damage in only 40 of the 109 struts in this truss (37\%). This will be explored further using frequency sensitivity in the following section.

This percentage is a function of the boundary conditions and would be different for a free-free truss, as would be the case for a structure in orbit. For comparison, Figure 5 shows the $50 \%, 75 \%$, and $95 \%$ cutoffs for the free-free case. The 95\% range for the free-free truss includes 56 members. The highly strained members are also more distributed throughout the structure.

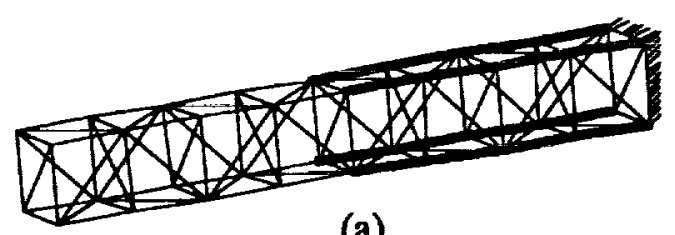

(a)

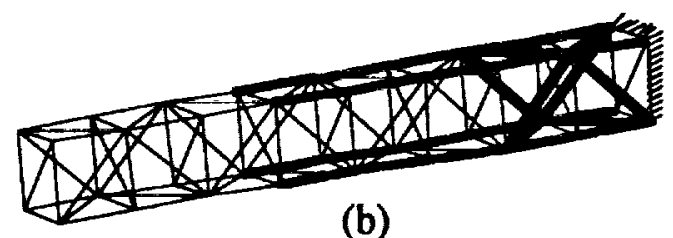

(b)

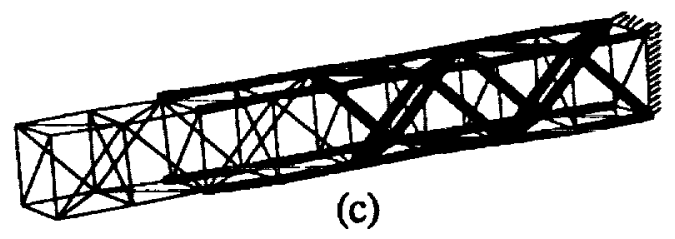

Figure 4. Strain energy for modes 1 through 6. Cantilevered boundary conditions

(a) $50 \%$ of Total - 161109 elements

(b) $75 \%$ of Total - 281109 elements

(c) $95 \%$ of Total - 401109 elements

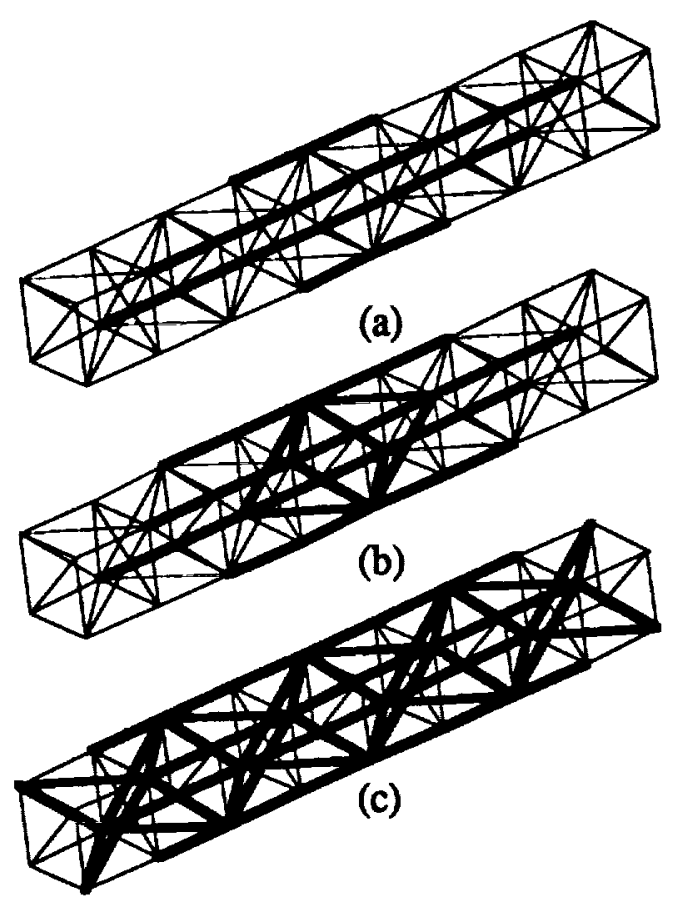

Figure 5. Strain energy for modes 1 through 6. Free-Free boundary conditions

(a) $50 \%$ of Total - 161109 elements

(b) $75 \%$ of Total - 28/109 elements

(c) $95 \%$ of Total - 561109 elements 


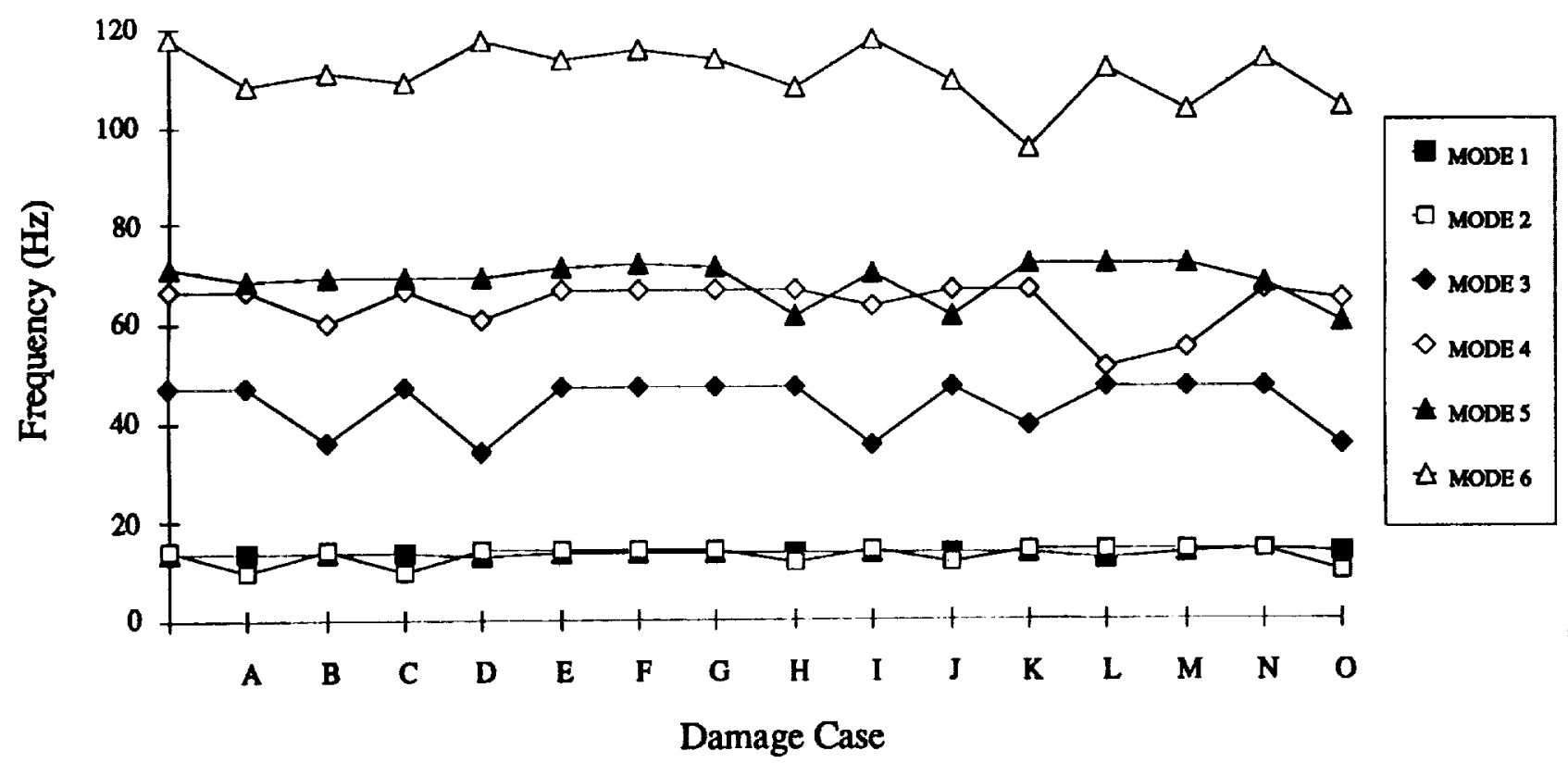

Figure 6. Frequency variations for eight bay damage cases.

\section{Enequency Sensitivity as a Damage Indicator}

Modal strain energy, as used in the previous section, is an indicator for damage detection feasibility and can be used to select appropriate modes to excite for improved damage detection performance.

Another process which can help determine mode selection is repeated analysis of the truss structure with each damage case modeled in turn. Frequency changes are then examined to select the modes which are influenced by the majority of the critical members.

Figure 6 shows how the frequencies change for $100 \%$ damage in each of the damage cases under consideration. It is important to note that there are several damage cases which cause modes 1 and 2 to switch order, as well as some instances of modes 4 and 5 switching order. In addition, several trends can be noted from such a plot. Large changes in the torsional modes can always be autributed to damage in a diagonal member. Significant changes in the bending modes can generally be attributed to longerons.

However, a more refined indicator that would allow examination of varying loss of stiffness in critical individual members would be useful. Researchers, such as Martinez ${ }^{5}$, have advocated using sensitivity analyses prior to system identification. Therefore, a second study examines the use of the sensitivity of frequencies and mode shapes to damage as a possible indicator for damage detection. Frequency sensitivities are calculated using the method developed by Fox and Kapoor 6

$$
\frac{\partial \lambda_{i}}{\partial p_{j}}=\frac{\{\phi\}_{i}^{T} \sum_{k=1}^{q} \frac{\partial\left[\mathrm{K}_{k}\right]_{j}}{\partial p_{j}}\{\phi\}_{i}-\lambda_{i}\{\phi\}_{i}^{T} \sum_{k=1}^{r} \frac{\partial\left[\mathrm{M}_{k}\right]}{\partial p_{j}}\{\phi\}_{i}}{\{\phi\}_{i}^{T}[\mathrm{M}]\{\phi\}_{i}}
$$

where $p_{j}$ is the $j$ th changing parameter and $\left[K_{k}\right]$ and $\left[M_{k}\right]$ are stiffness and mass matrices for the $k$ th element, respectively; and $q$ and $r$ are the total number of stiffness and mass elements within the structure, respectively. For the eight bay truss, each member is modeled using a rod element and the stiffness is linearly related to Young's modulus, E. Thus, in this study, Young's modulus for each member is selected as the changing parameter.

If we assume that the damage can be described as a loss of stiffness without changing the mass properties of the structure, equation (5) can be simplified to

$$
\frac{\partial \lambda_{i}}{\partial p_{j}}=\frac{\{\phi\}_{i}^{T} \frac{d\left[K_{j}\right]}{d p_{j}}\{\phi\}_{i}}{\{\phi\}_{i}^{T}[\mathbf{M}]\{\phi\}_{i}}
$$

Introducing the orthogonality relationships of equation (3) into equations (4) and (6), the strain energy distribution for member $j$ in mode $i$ and the sensitivity of the ith eigenvalue to changes in stiffness of member $j$ are related: 


$$
\begin{aligned}
& \frac{\partial \lambda_{i}}{\partial p_{j}}=\{\phi\}_{i}^{T} \frac{d\left[\mathbf{K}_{j}\right]}{d p_{j}}\{\phi\}_{i} \\
& S E_{j i}=\frac{\{\phi\}_{i}^{T}\left[K_{j}\right]\{\phi\}_{i}}{\lambda_{i}} \times 100 \%
\end{aligned}
$$

The frequency sensitivity, however, allows us to examine partial damage for situations of less than $100 \%$ stiffness loss.

Equation (7a) is only accurate for small changes in stiffness. In order to examine the full range of damage possibilities (from 0 to $100 \%$ stiffness loss) without the computational burden of repeated finite-element analyses, an iterative sensitivity algorithm was developed. At each iteration of the algorithm equation (7a) is used to determine the change in frequency with updated mode shape vectors. The mode shapes are updated using Nelson's eigenvector derivative technique? ${ }^{7}$. The use of the eigenvector derivative serves two purposes. First, it avoids the need to re-solve the eigenvalue equation at each step. Second, it provides further insight into the effect that damage has on the modal properties, namely the change in the mode shape vectors. In this paper, only the results from the frequency sensitivity studies will be presented.

As an example, Figure 7 shows percent changes in the first six natural frequencies of the structure as the stiffness of longeron number 35 (Damage Case $\mathrm{H}$ ) on the third bay from

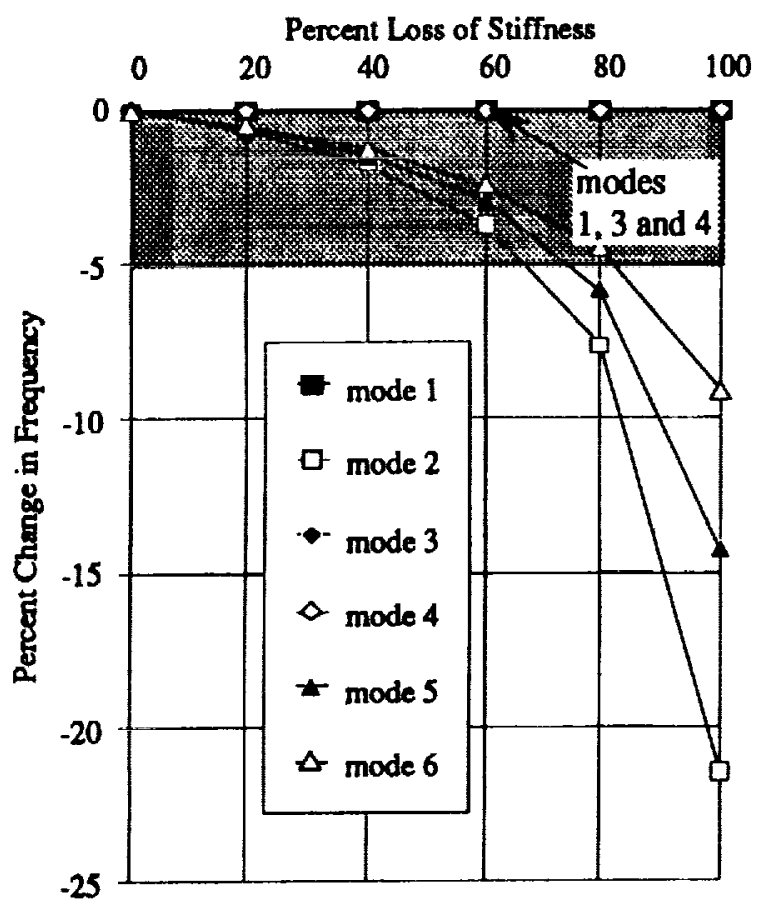

Figure 7. Frequency variation for progressive damage in longeron 35 . the cantilevered end is reduced due to damage. Mode 2, which is the second of the first bending mode pair, is most sensitive to the stiffness loss. Modes 5 and 6 , which are the second bending and the first axial mode, respectively, are also influenced by the damage. However, modes 1,3 , and 4 are not influenced by the damage at the longeron at all. The plane of bending motion is orthogonal for the paired modes, i.e.,modes 1 and 2, and modes 4 and 5. Modes 1 and 4 are not influenced by this damaged element while modes 2 and 5 are. Mode 3, which is the first torsional mode, strains predominantly the diagonals and is affected only slightly by the stiffness changes in the longerons.

This sensitivity study also indicates that only modes 2,5 and 6 would be useful for damage detection for case $H$. This confirms the results of the strain energy study. More importantly, this study indicates that damage detection is unlikely for a small loss of stiffness in this longeron. The shaded portion of the plot indicates that if the frequency measurement accuracy is considered to be $\pm 5 \%$, then no measurable change in frequency for any mode would be apparent unless the stiffness loss of longeron 35 exceeded $65 \%$.

As a second example, Figure 8 presents the sensitivity study for the the case of a diagonal which only affects the torsional mode.

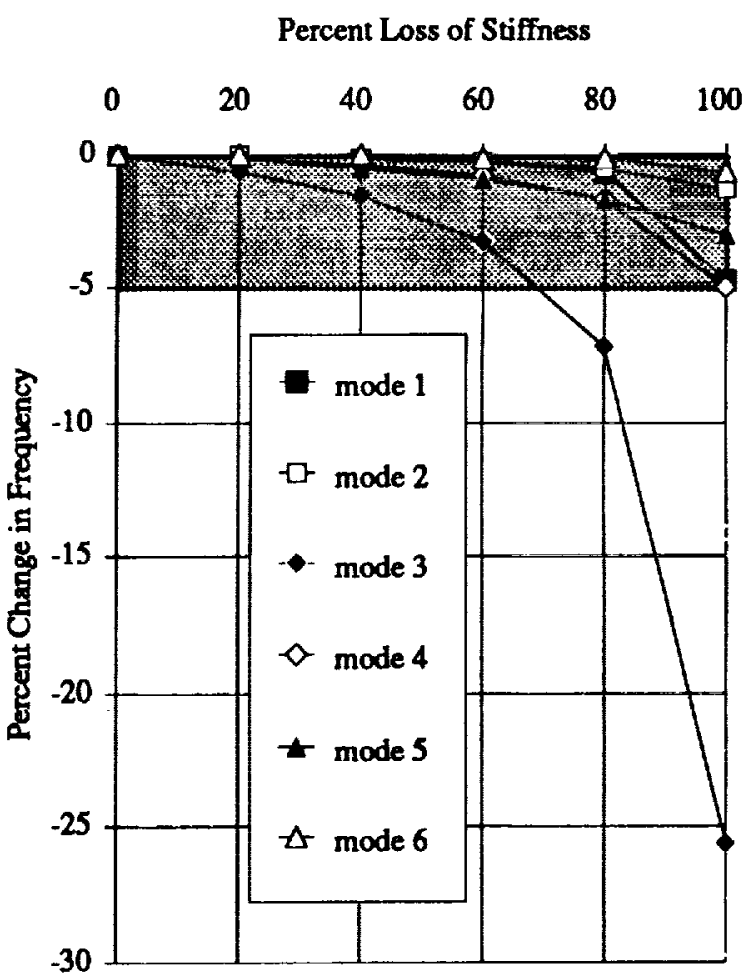

Figure 8. Frequency variation for progressive damage in diagonal 99 
A 70\% loss of stiffness in the member would be necessary before the damage would be apparent in the frequency (assuming 5\% measurement error). If test results for the torsional mode are not available, more accurate frequency measurements of other modes would be required to make damage detection of this member feasible.

Finally, as an extreme example, Figure 9 shows the frequency sensitivity for a bulkhead diagonal between bays 2 and 3 (damage Case F). Notice that only the axial mode is affected by the damage, and even at $100 \%$ damage, the change in frequency is less than $3 \%$. With only six measured modes it is impossible to detect damage in this member without additional instrumentation.

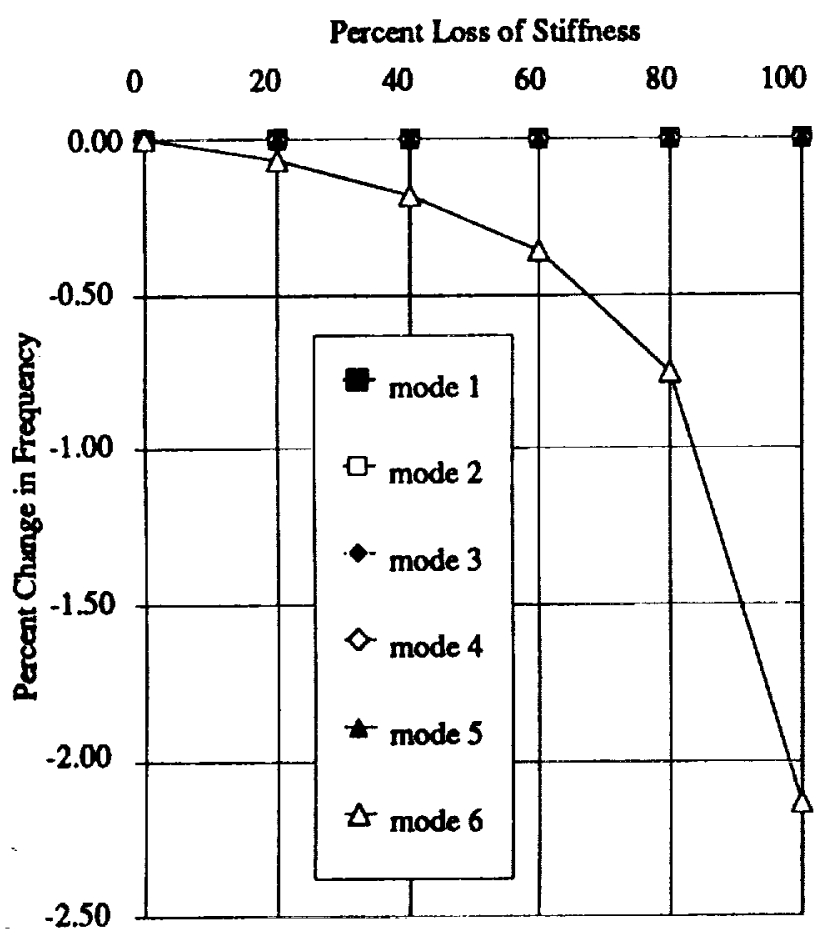

Figure 9. Frequency variation for progressive damage in diagonal 75.

Frequency sensitivity studies for the range of stiffness loss in individual members are useful for mode selection and as a more refined indication of detection feasibility. These studies, when performed for critical members, indicate critical modes for the test design, but also suggest performance limitations which will accompany measurement accuracy. These studies therefore give guidance for the selection of the modal property extraction method and the test instrumentation, as well.

\section{Mode Shape Sensitivity and Variations Due to Damage}

The sensitivity of the mode shapes to damage can be explored using the eigenvector derivatives. Using the mode shape sensitivities is more complicated than the frequency sensitivities since the mode shapes and sensitivity coefficients are both vectors as opposed to scalars. In this section the equations for the eigenvalue sensitivities are presented, as well as a discussion of the effect of damage on the mode shapes.

The governing equation for the eigenvector derivatives is found by differentiating (2) with respect to the parameter $p_{j}$

$$
\left([\mathbf{K}]-\lambda_{i}[\mathbf{M}]\right) \frac{\partial\{\phi\}_{i}}{\partial p_{j}}=-\left(\frac{\partial[\mathrm{K}]}{\partial p_{j}}-\frac{\partial \lambda_{i}}{\partial p_{j}}[\mathbf{M}]-\lambda_{i} \frac{\partial[\mathrm{M}]}{\partial p_{j}}\right)\{\phi\}_{i}
$$

The matrix $\left([K]-\lambda_{i}[M]\right)$ is singular since $\lambda_{i}$ is an eigenvalue. In order to solve for the eigenvector derivative, Nelson represents it as

$$
\frac{\partial\{\phi\}_{i}}{\partial p_{j}}=[P]+c\{\phi\}_{i}
$$

where $[\mathrm{P}]$ is a particular solution found by identifying the largest absolute value in the vector $\{\phi\}_{i}$ and constraining the derivative of that component to zero. The value of the constant $\mathrm{c}$ is then found by differentiating the first of the orthogonality equations (3) and substituting (9) into the result and simplifying

$$
c=-\{\phi\}_{i}^{T}[\mathrm{M}][P]-\frac{1}{2}\{\phi\}_{i}^{T} \frac{\partial[\mathrm{M}]}{\partial p_{j}}\{\phi\}_{i}
$$

The mode shape sensitivity coefficients can be plotted for each node in each mode as the stiffness decreases from $100 \%$ to $0 \%$. Large changes in the coefficients at nodes near the damage site have been seen and this is being explored as a possible damage detection tool.

A measure of the differences between two modal vectors can be calculated using the Modal Assurance Criterion (MAC) ${ }^{8}$, defined as

$$
M A C=\sqrt{\frac{\left(\{\phi\}_{c}^{T}\{\phi\}_{n}\right)^{2}}{\left(\{\phi\}_{c}{ }^{T}\{\phi\}_{c}\right)\left(\{\phi\}_{n}{ }^{T}\{\phi\}_{n}\right)}}
$$

where the subscript $c$ indicates corrupted, or damaged, modes, and the subscript $n$ indicates normal modes. If two modal vectors are identical, the MAC value is expected to be unity. If the two modes are orthogonal, the MAC will have a value of zero. The MAC is commonly used to correlate test and analysis modes.

The modes obtained from the tests of the undamaged structure were compared to the normal modes from the original finite-element model. Table 4 presents these results. The columns represent the first six test modes, 
while the rows represent the corresponding analysis modes. In practice, off-diagonal terms less than 0.1 indicate an acreptable test.

Table 4 MAC matrix for undamaged test vs. undamaged analysis

\begin{tabular}{ccccccc}
\hline & 1 & 2 & 3 & 4 & 5 & 6 \\
1 & 0.995 & 0.000 & 0.000 & 0.023 & 0.000 & 0.006 \\
2 & 0.000 & 0.995 & 0.002 & 0.000 & 0.046 & 0.003 \\
3 & 0.000 & 0.000 & 0.941 & 0.000 & 0.000 & 0.014 \\
4 & 0.035 & 0.000 & 0.000 & 0.964 & 0.000 & 0.002 \\
5 & 0.000 & 0.041 & 0.002 & 0.001 & 0.982 & 0.008 \\
6 & 0.012 & 0.009 & 0.023 & 0.006 & 0.016 & 0.979 \\
\hline
\end{tabular}

Tables 5 and 6 demonstrate that the modes obtained from a damage test are significantly different from those of the undamaged structure. Table 5 compares modes from damage case $\mathrm{H}$ with the undamaged modes. Notice that modes 1 and 2, as well as modes 4 and 5 have switched order, as indicated by zero diagonal terms and non-zero offdiagonal terms.

Table 5 MAC matrix for damage case $\mathrm{H}$ test modes vs undamaged analysis modes

\begin{tabular}{ccccccc}
\hline & 1 & 2 & 3 & 4 & 5 & 6 \\
1 & 0.000 & 0.994 & 0.000 & 0.000 & 0.005 & 0.000 \\
2 & 0.992 & 0.000 & 0.000 & 0.000 & 0.000 & 0.003 \\
3 & 0.000 & 0.000 & 0.946 & 0.000 & 0.000 & 0.000 \\
4 & 0.000 & 0.005 & 0.000 & 0.000 & 0.957 & 0.000 \\
5 & 0.019 & 0.000 & 0.000 & 0.932 & 0.000 & 0.057 \\
6 & 0.002 & 0.000 & 0.000 & 0.040 & 0.000 & 0.894 \\
\hline
\end{tabular}

Table 6 compares modes from damage case $F$ with the undamaged modes. Notice that the diagonal terms, with the exception of mode 6, are all almost equal to or higher than the equivalent term in Table 4, the undamaged test vs undamaged analysis. The MAC value for mode 6 is considerably lower than the one in table 4 indicating that there may indeed be damage. Recall that the frequency changes for this damage case were too small to indicate damage. The mode shape comparison, on the other hand indicates the possibility of damage.

Table 6 MAC matrix for damage case F test modes vs undamaged analysis modes

\begin{tabular}{ccccccc}
\hline & 1 & 2 & 3 & 4 & 5 & 6 \\
1 & 0.998 & 0.008 & 0.001 & 0.027 & 0.003 & 0.012 \\
2 & 0.010 & 0.996 & 0.007 & 0.000 & 0.046 & 0.006 \\
3 & 0.000 & 0.000 & 0.938 & 0.000 & 0.000 & 0.008 \\
4 & 0.049 & 0.000 & 0.000 & 0.960 & 0.000 & 0.006 \\
5 & 0.005 & 0.032 & 0.008 & 0.001 & 0.986 & 0.008 \\
6 & 0.012 & 0.009 & 0.019 & 0.002 & 0.031 & 0.912 \\
\hline
\end{tabular}

Although the mode shape variations explored in this work are somewhat qualitative they do indicate that mode shape variations due to damage may be more sensitive than frequencies and may provide a better indicator of damage. Use of the mode shape sensitivities as an indicator of the existence of damage will be pursued in future studies.

\section{Influence of Modelling and Measurement Accuracy on Erequencies and Mode Shapes}

In the previous sections, the assumption was made that the measured modes and frequencies were known to within $\pm 5 \%$ of the actual values. This assumption was used to determine the ability to locate damage. In the past, researchers have used similar assumptions to create simulated measured data for use in developing identification methods and for damage location. In this section, effects of modelling and measurement errors on the modal data are examined and compared with the modes and frequencies from simulated models. By understanding the true effects of damage, modelling errors, and measurement errors on the modes and frequencies, accurate simulations can be created for use in damage location studies.

\section{Erequency measurement accuracy}

Many researchers use simulated test data to explore the effects of noise and measurement errors on their identification algorithms. Smith ${ }^{9}$, among others, has added random errors to the normal modes predicted for the damaged structure. Reasonable performance of one class of identification methods (optimal update methods) is expected if added random errors are less than $\pm 5 \%$.

For comparison, Table 7 includes the frequencies and percent differences between the analysis and the test for the undamaged eight bay structure. The differences between test and analysis frequencies for the undamaged truss are less than 5.5\%. Table 7 also includes the test results for two of the previous example damage cases (Case $\mathrm{H}$ - longeron member 35 and Case F - diagonal member 75).

\section{Mode shape yariations.}

In past work, possible variations that exist in the mode shapes due to modelling error, the testing process or errors introduced by the modal identification method have not been adequately examined. A study of variations in mode shapes was undertaken to address this shortcoming. Normal mode shapes from the finite element model of the eight bay truss are compared to modes from tests of the actual truss structure, to normal modes which are corrupted with random errors, and finally to modes from a finite-element model with random errors in the geometry. With these results, more realistic modal data can be specified in simulated 
Table 7 Frequency comparison for model, test, damage and geometry variations

\begin{tabular}{|c|c|c|c|c|c|c|c|c|c|c|c|}
\hline & \multicolumn{3}{|c|}{ UNDAMAGED } & \multicolumn{3}{|c|}{ DAMAGE CASE H } & \multicolumn{3}{|c|}{ DAMAGE CASE F } & \multicolumn{2}{|c|}{$\begin{array}{l}\text { MODIFIED } \\
\text { GEOMETRY }\end{array}$} \\
\hline MODE & FEM & TEST & $\begin{array}{c}90 \\
\text { Difference }\end{array}$ & FEM & TEST & $\begin{array}{c}\% \\
\text { Difference }\end{array}$ & FEM & TEST & $\begin{array}{c}\$ \\
\text { Difference }\end{array}$ & $\begin{array}{c}1 \mathrm{x} \\
\text { tolerance }\end{array}$ & $\begin{array}{c}\% \\
\text { Difference }\end{array}$ \\
\hline 1 & 14.005 & 13.969 & 0.257 & 14.007 & 13.972 & 0.250 & 14.007 & 13.981 & 0.186 & 14.026 & -0.1 \\
\hline 2 & 14.521 & 14.597 & -0.052 & 11.415 & 11.531 & -1.016 & 14.523 & 14.557 & -0.234 & 14.530 & -0.1 \\
\hline 3 & 46.615 & 48.431 & -3.896 & 46.672 & 47.859 & -2.543 & 46.646 & 48.623 & -4.238 & 46.495 & 0.3 \\
\hline 4 & 66.238 & 64.306 & 2.917 & 66.384 & 65.226 & 1.744 & 66.415 & 65.012 & 2.113 & 66.276 & -0.01 \\
\hline 5 & 71.328 & 67.440 & 5.451 & 61.396 & 59.997 & 2.279 & 71.463 & 68.256 & 4.488 & 71.276 & 0.01 \\
\hline 6 & 117.949 & 112.795 & 4.369 & 107.384 & 104.963 & 2.255 & 115.370 & 109.379 & 5.193 & 118.007 & -0.05 \\
\hline
\end{tabular}

verification studies of system identification techniques. This allows for a more realistic assessment of identification techniques at an earlier stage in their development.

In order to evaluate how simulated test modes compare to real test modes, each of the normal modes was corrupted by adding a uniformly distributed random vector $\{R\}$, with values between \pm 0.05 times the maximum value of the analytical mode, to the mode shape coefficients.

$$
\{\phi\}_{i}{ }^{c}=\{\phi\}_{i}+\{R\}
$$

Table 8 shows that the simulated modes are qualitatively different from real test modes. The diagonal terms are almost unity and the off-diagonal terms are all higher than the corresponding terms in Table 4 . This suggests that simulating test modes by adding $\pm 5 \%$ error to the normal modes may be a quick way to verify that an algorithm will work with corrupted data, but is not an accurate simulation of real test data.

Table 8. MAC matrix for normal modes vs. normal modes with $\pm 5 \%$ random error

\begin{tabular}{ccccccc}
\hline & 1 & 2 & 3 & 4 & 5 & 6 \\
1 & 0.998 & 0.036 & 0.001 & 0.070 & 0.026 & 0.033 \\
2 & 0.006 & 0.999 & 0.001 & 0.003 & 0.055 & 0.033 \\
3 & 0.001 & 0.042 & 0.998 & 0.004 & 0.012 & 0.037 \\
4 & 0.066 & 0.035 & 0.002 & 0.998 & 0.020 & 0.032 \\
5 & 0.004 & 0.029 & 0.003 & 0.001 & 0.998 & 0.023 \\
6 & 0.002 & 0.036 & 0.001 & 0.003 & 0.016 & 0.998 \\
\hline
\end{tabular}

\section{Modelling and manufacturing errors}

There has been some concem from researchers that analytical model updating using test data will not be a practical method for damage location because errors in modelling or changes in the structure over time will cause the measured modes to be incompatible with the analytical model. In addition to examining the effect of errors in measurement, the study also addressed the possibility of either errors in manufacturing, or errors in modelling. A random error was added to the lengths of the eight bay struts to simulate enrors in modelling or errors in manufacturing. Error levels representing $1 \mathrm{x}, 5 \mathrm{x}$ and $10 \mathrm{x}$ the manufacturers tolerance for the lengths of the hybrid model struts were examined. Table 7 includes the frequency results for the first of these cases. Figure 10 is an example of the three error vectors that were added to the $\mathrm{x}, \mathrm{y}$ and $\mathrm{z}$ coordinates for the 36 nodes.

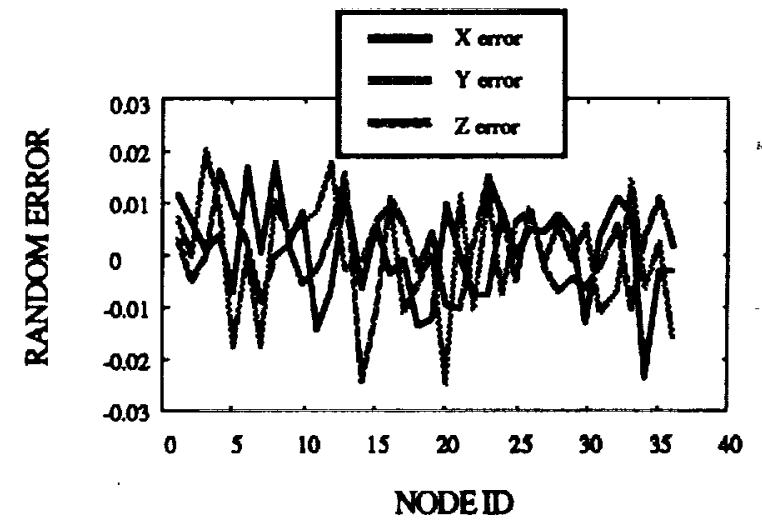

Figure 10. Random error applied to geometry.

Table 9 shows the MAC matrix between the normal modes and the modes that result from an eigenvalue solution using the adjusted stiffness matrix with the random error added to the geometry.

Table 9 MAC Matrix for maximum manufacturers error in geometry

\begin{tabular}{ccccccc}
\hline & 1 & 2 & 3 & 4 & 5 & 6 \\
1 & 0.999 & 0.001 & 0.000 & 0.005 & 0.000 & 0.000 \\
2 & 0.001 & 0.999 & 0.000 & 0.000 & 0.006 & 0.000 \\
3 & 0.000 & 0.000 & 1.000 & 0.000 & 0.000 & 0.000 \\
4 & 0.004 & 0.000 & 0.000 & 1.000 & 0.000 & 0.000 \\
5 & 0.000 & 0.005 & 0.000 & 0.000 & 1.000 & 0.000 \\
6 & 0.000 & 0.000 & 0.000 & 0.000 & 0.000 & 1.000 \\
\hline
\end{tabular}

Five times the manufacturers tolerance has to be added to the geometry before any significant changes changes in the MAC appears as shown in Table 10 . This suggests that 
the modes and frequencies from a test will be useful for damage location as long as the model of the structure is reasonably accurate. Further quantification of the modelling accuracy will be attempted in the future.

Table 10 MAC matrix for $5 \times$ maximum manufacturers error in geometry

$\begin{array}{ccccccc} & 1 & 2 & 3 & 4 & 5 & 6 \\ 1 & 0.999 & 0.025 & 0.000 & 0.005 & 0.000 & 0.000 \\ 2 & -0.025 & 0.999 & 0.000 & 0.000 & 0.006 & 0.000 \\ 3 & 0.000 & 0.000 & -0.999 & -0.008 & 0.000 & 0.000 \\ 4 & 0.004 & 0.000 & 0.000 & 1.000 & 0.000 & 0.000 \\ 5 & 0.000 & 0.005 & 0.000 & 0.000 & 1.000 & 0.000 \\ 6 & 0.000 & 0.000 & 0.000 & 0.000 & 0.000 & 0.999\end{array}$

More work needs to be done to quantify the expected errors and differences between test and analysis mode shapes in order to simulate test data more accurately and thereby improve the robustness of modal identification methods. With statistics on the variations that occur in the modal identification, more appropriate simulation procedures can be developed.

\section{Summary and Conclusions}

In this paper, expectations for the ability to locate damage are evaluated through the use of indicators which reflect the limitations of modal data which will be used in the identification process. A pre-test analysis of strain energy distribution defines where damage location is likely and where it is unlikely. Mode selection and test design for damage detection are supported by strain energy analysis as well. Frequency sensitivity requires more computational effort, but provides a conservative indicator of ability to detect damage, even for partial damage to members. Mode shape differences that result from damage to the truss structure are significant, while differences that result from geometric variations and simulated random errors are not, indicating that mode shapes contain useful information to supplement frequency information for the damage location problem. Inaccuracy of the measured modes may also limit the damage detection capability. Frequency and mode shape sensitivity information can be utilized to provide insight into the measurement accuracy requirements. System identification techniques for damage location should now be evaluated and verified within expectations and limitations of the modal data. However, more work needs to be done to quantify the relationship between mode shape sensitivities and mode shape measurement errors and the ability to locate damage.

\section{Acknowledgements}

Special thanks to Dr. Joe Eisley, of the University of Michigan, for his help with the sensitivity runs, and Alan Stockwell, of Lockheed Engineering and Sciences Co., for his valuable suggestions.

\section{References}

1. Kashangaki, T. A.-L., "On-Orbit Damage Detection and Health Monitoring of Large Space Trusses - Status and Critical Issues," 32nd AIAA SDM Conference, Baltimore, Md. April 8-10, 1991.

2. Vold, H., Kundrat, J., Rocklin, G.T., Russell, R., "A Multi-input Modal Estimation Algorithm for Mini-computers", SAE Paper No. 820194, 1982.

3. Hajela and Soeiro, "Recent Developments in Damage Detection Based on System Identification Methods," Structural Optimization, Vol. 2, 1990, pp 1-10.

4. Chen, J.C. and Garba, J.A., "On Orbit Damage Assessment for Large Space Structures ", AIAA Journal, Vol. 26, No. 9, September 1988.

5. Martinez, D.R, Red-Horse, J.R., and Allen, J.J.,"System Identification Methods for Dynamic Structural Models of Electronic Packages," 32nd AIAA SDM Conference, Baltimore, Md. April 8$10,1991$.

6. Fox, R. L. and Kapoor, M. P., "Rates of Change of Eigenvalues and Eigenvectors," AIAA Joumal. Vol. 6, Dec. 1968, pp. 2426-2429.

7. Nelson, R.B., "Simplified Calculation of Eigenvector Derivatives," AIAA Joumal, Vol. 14, No. 9, 1976, pp. 1201-1205.

8. Ewins, D.J., Modal Testing: Theory and Practice. Research Studies Press, 1984.

9. Smith, S.W., "Damage Detection and Location in Large Space Trusses," Doctoral Dissertation, Virginia Polytechnic Institute and State University; Blacksburg, Virginia, 1988. 
Public reporting burden for this collection of information is estimated to average I hour per response, including the time for reviewing instructions, searching existing dato sources. gathering and maintaining the data needed, and completing and reviewing the coliection of information. Send comments regarding this burden estimate or any other aspect of this Davis Highway. Suite 1204. Arlington, VA 22202.4302, and to the Office of Management and Budget. Paperwork Reduction Project (0704-0 188), Washington, DC 20503.

\begin{tabular}{|l|l|l}
\hline 1. AGENCY USE ONLY (Leave blank) & $\begin{array}{l}\text { 2. REPORT DATE } \\
\text { February } 1992\end{array}$ & $\begin{array}{c}\text { 3. REPORT TYPE AND DATES COVERED } \\
\text { Technical Memorandum }\end{array}$
\end{tabular}

4. TITLE AND SUBTITLE

Underlying Modal Data Issues for Detecting Damage in

Truss Structures

\section{AUTHOR(S)}

Thomas A-L. Kashangak1, Suzanne Weaver Smith, and Tae W. Lim

\section{PERFORMING ORGANIZATION NAME(S) AND ADDRESS(ES)}

NASA Langley Research Center

Hampton, VA 23665-5225

\section{SPONSORING/MONITORING AGENCY NAME(S) AND ADDRESS(ES)}

National Aeronautics and Space Administration

Washington, DC 20546-0001

\section{FUNDING NUMBERS}

WU 590-14-31-01
8. PERFORMING ORGANIZATION REPORT NUMBER

\section{SUPPLEMENTARY NOTES}

Kashangaki: Langley Research Center, Hampton, VA; Smith: University of Kentucky, Lexington, KY; Lim: Lockheed Englneering \& Sciences Co., Hampton, VA

To be presented at the 33rd Structures, Structural Dynamics, and Materials Conf. Dallas, TX, Apr11 13-17, 1992

12.. DISTRIBUTION/AVAILABILITY STATEMENT

12b. DISTRIBUTION CODE

Unclassified-Unlimited

Subject Category 39

\section{ABSTRACT (Maximum 200 words)}

Independent of the modal identification techniques employed for damage detection, use of measured modal data limits the expectations for damage location. These limitations are examined using the distribution of modal strain energy and the sensitivity of the frequency and mode shapes to structural stiffness changes. For given measured modal information of specified accuracy, this examination reveals the following:

(1) damage detection is feasible for members that contribute significantly to the strain energy of the measured modes, (2) the modes.which are most effective in detecting damage to certain critical members can be identified and (3) a relationship can be drawn between the accuracy of the measured modes and frequencies and damage detection feasibility.

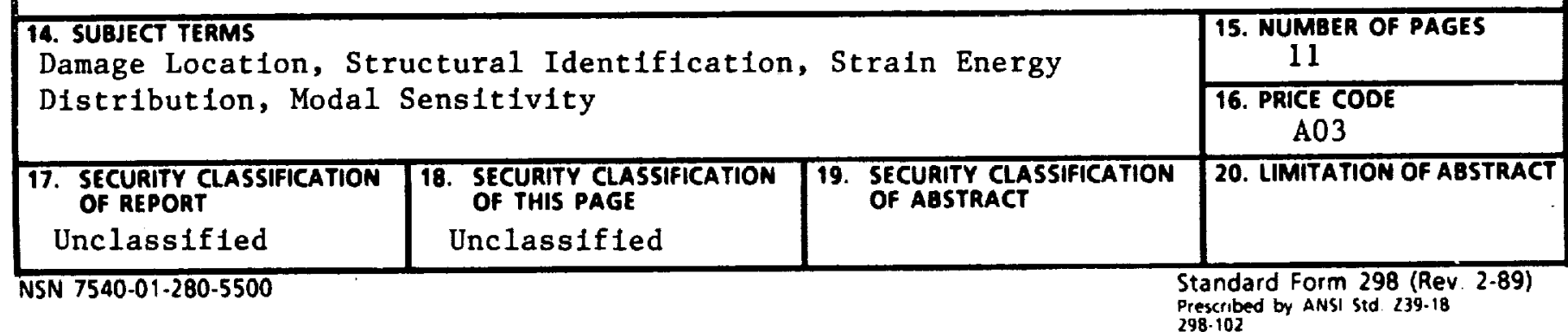


